\title{
Zum Einsatz von Kinomobilen für die Kirche in Entwicklungsländern
}

\author{
von Wilhelm Herzog
}

\section{Fragestellung}

Die Frage nach der Effizienz bestimmter missionarischer und pastoraler Hilfsmittel wird hier, abgesehen von der theologischen Problematik im engeren Sinne, als kommunikationstheoretische und religionspädagogische Frage nach überprüfbaren Wirkungen von zielorientierten kirchlichen Aktivitäten verstanden. Die in Briefen und Tagungsberichten ${ }^{1}$ aus Entwicklungsländern immer wieder durchklingende optimistische Feststellung, daß Kinomobile (Mobile Film Units) eine weitaus effektivere Verkündigung und Entwicklungshilfe als andere audiovisuelle oder rein verbale Kommunikationsformen ermöglichten, soll kritisch hinterfragt werden. Dabei ist die Beschränkung der Blickrichtung auf Kinomobile methodischer Art. Die Arbeit auf diesem Sektor erscheint im Vergleich zu Presse, Rundfunk, Predigt, Katechese, Literatur oder Sozialarbeit noch relativ überschaubar; sie ist weniger mit Motiven der Institutionssicherung befrachtet, allerdings oft mit großen finanziellen Aufwendungen für das einzelne Projekt verbunden.

Die Klärung der Problematik kann sich bisher weder auf exaktes statistisches Material noch auf wissenschaftliche Beobachtungsreihen stützen. Es scheint sich aber bei den für Mission oder Seelsorge verantwortlichen Personen bzw. Institutionen in Entwidklungsländern ein Trend zum Kinomobil zu entwickeln. Gerade wegen dieser sich ausweitenden Praxis seien hier einige theoretische Gesichtspunkte vorgelegt, die den notwendigen Regelkreis von Theorie und Praxis in Gang setzen könnten.

\section{Zur bisherigen Praxis}

Nach einer Auskunft des Catholic Media Council in Aachen² waren bis Ende 1971 allein in Asien wenigstens zwölf (davon neun in Indien) der im wesentlichen aus Transportfahrzeug, Vorführgeräten und Generator bestehenden Kinomobile im Einsatz. Klimatische, geographische und organisatorische Schwierigkeiten brachten es mit sich, daß sich die Arbeit dieser Einheiten oft nur auf wenige Monate im Jahr und auf ein relativ begrenztes Einsatzgebiet erstreckte. Das Personal dieser fahrbaren Kinos bestand in den meisten Fällen aus dem Fahrer, der im Idealfall auch als Mechaniker und Vorführer fungierte, und einem Priester oder Katecheten, die sich oft nebenamtlich der Filmarbeit widmeten. Für Schulung und Erfahrungsaustausch stehen in Afrika und Asien bisher keine interdiözesanen oder auch interkonfessionellen spezialisierten Trainingszentren zur Verfügung. Deswegen ist das Personal der Kinomobile oft auf sich selbst angewiesen, und die Ausbildung läßt nach Ansicht von Fachleuten zu wünschen übrig. Auf Charakter und Ursache dieses Mangels kann hier nicht näher eingegangen werden.

Die Zahl der Veranstaltungen pro Jahr und Kinomobil schwankt zwischen 50 und 100; in Einzelfällen, etwa bei Einführung eines neuen religiösen Films (wie "Son

Wilhelm Herzog, Diplom-Theologe, ist ständiger Mitarbeiter des "Catholic Media Council“ in Aachen und Herausgeber der Bibliographie "Church and Communication in Developing Countries" (Paderborn 1973). 
of $\mathrm{Man}^{\alpha}$ (oder anläßlich religiöser Wochen stieg die Frequenz auf 100 bis 200 Vorführungen innerhalb weniger Wochen. Ein Vergleich mit der Einsatzquote staatlich betriebener Kinomobile kann wegen der unterschiedlichen Bedingungen beider Unternehmen und wegen des spärlichen Datenmaterials nicht gezogen werden. Von kirchlichen und staatlichen Stellen in Entwicklungsländern wird aber immer wieder festgestellt, daß die vorhandenen Mittel noch weitaus rationeller als bisher eingesetzt werden könnten.

Für die einzelne Veranstaltung hat sich vielfach die Praxis durchgesetzt, das Programm nicht nur aus religiösen, sondern auch aus belehrenden und unterhaltenden Filmen zu gestalten, wobei die Auswahl bei äußerst begrenztem Angebot kirchlicher oder staatlicher Verleihorganisationen mehr oder weniger zufällig zustande kommt. An Motiven (Bibelthemen, Rosenkranz, Ereignisse der Weltkirche) und Gestaltungsformen (Spielfilm, Dokumentarfilm, Dokumentarspiel) westlicher Prägung ausgerichtet, wird der religiöse Teil des Programms von den Zuschauern zuweilen als Pflichtübung angesehen, dem der unterhaltende Teil - meist ebenso sehr westlicher Provenienz und daher ebenso problematisch - als eigentliches Erlebnis folgt oder vorausgeht. Über den möglichen oder tatsächlichen Beitrag der einzelnen Filmkategorien zur Gesamtzielsetzung solcher Veranstaltungen ist damit aber noch nichts gesagt. Bemerkenswert ist immerhin die Wertung der meisten in der Filmarbeit engagierten Priester und Missionare, daß die Filmabende bei der einheimischen Landbevölkerung starke, religiös zu charakterisierende Emotionen hervorrufen und daß die Gesprächsbereitschaft gegenüber Vertretern der Kirche von seiten der Christen und Nichtchristen zunimmt. Worin sich aber der Effekt eines Filmabends von dem anderer, etwa sozial-karitativer Wirksamkeit, unterscheidet, bleibt unklar, zumal, wenn in regionalen Arbeitsgesprächen die Frage nach der Nacharbeit zum Film oft gar nicht berührt, von einigen aber mit dem Hinweis auf Krankenpflege und Gottesdienst pauschal beantwortet wurde. ${ }^{3}$

\section{Zur kommunikationstheoretischen Grundlegung}

Man könnte einwenden, daß die obige Skizzierung die Wirklichkeit verfehle und $\mathrm{da}$ die Verantwortlichen der lokalen, einheimischen Kirchen doch am besten die Wirksamkeit der von ihnen eingesetzten Mittel beurteilen könnten. Dazu ist zu sagen, daß die Effizienz von Verkündigung oder Entwicklungshilfe durch Film vielfach eher vorausgesetzt als exakt beschrieben oder gar nachgewiesen wird. Es geht hier deswegen darum, die Perspektiven, unter denen die Praxis zu sehen ist, und ihre Zielvorstellungen zu präzisieren. Um einen derartigen Ansatz zu gewinnen, scheint es sinnvoll, von der 1971 erschienenen Unesco-Studie von Peter Hopkinson "The rôle of film in development ${ }^{\text {" }}$ auszugehen, die in kritischer Absetzung von der gegenwärtigen Praxis der Kinomobile in Entwicklungsländern zu folgenden Ergebnissen kommt:

\section{Inhalt und Form der Filme}

a) Die von Kinomobilen gezeigten Filme müssen auf ein ländliches Publikum zugeschnitten sein. Kopien von $35-\mathrm{mm}$-Filmen, die für ein städtisches Publikum gedreht wurden, verfehlen bei der vorwiegend analphabetischen Landbevölkerung ihre Wirkung. ${ }^{5}$

b) Soweit es die technischen und finanziellen Mittel erlauben, sollen die für Kinomobile bestimmten Filme im Entwicklungsland selbst unter Berücksichtigung der regionalen soziokulturellen Bedingungen produziert werden. ${ }^{6}$ 
c) Die Hauptaussage eines Films, vor allem des Dokumentarfilms, muß im Bild deutlich werden. Der gesprochene Kommentar und die vom Wort her bedingte Logik eines Films werden von dem genannten Publikum nicht adäquat erfaßt. Es erlebt den Film eher nach Art von Traumbildern als in der geordneten Sequenz von Szenen. ${ }^{7}$

d) Die von Kinomobilen gezeigten Filme sollen sich am individuell gestalteten, persönlich ansprechenden Fernsehprogramm orientieren, das sich ebenso vom Schema der Radioprogramme wie auch des kommerziellen Spiel- oder Dokumentarfilms emanzipiert hat. Es hängt vom Stand der Infrastruktur des jeweiligen Landes ab, ob der Effekt des bewegten Bildes durch Film, Fernsehen oder Videorecorder angezielt werden kann. ${ }^{8}$

\section{Arbeit der Kinomobile}

a) Der engere Rahmen der Vorführung muß vom Personal der Kinomobile und den lokalen Entwicklungsdiensten gemeinsam gestaltet werden. Gerade letztere können die im Film gezeigte Welt, den Informationsgehalt des Films und seine Anregungen zu Innovationen am elesten den Rezipienten auf dem Lande, mit denen sie Tag für Tag zusammenleben, vermitteln. Der Film wird gleich den anderen Massenmedien nur in Verbindung mit interpersonaler Kommunikation auf unterster Ebene wirksam. $^{\theta}$

b) Für den weiteren Rahmen der Kinomobil-Einsätze ist die Forderung nach einem Strukturplan zu erheben. Dieser Plan soll die verschiedenen publizistischen Aktivitäten in einem Land koordinieren und Schwerpunkte setzen. Die Arbeitsweise der Kinomobile, die Qualifikation des Personals und die Wartung der technischen Ausstattung sind regelmäßig zu kontrollieren. ${ }^{10}$

\section{Zur religionspädagogischen Grundlegung}

Gaston Roberge umreißt den pädagogischen Aspekt der Filmarbeit in Entwicklungsländern folgendermaßen: „Eine Filmarbeit, die sich ihrer Verantwortung als Massenmedium bewußt ist, sollte sich ins Erziehungssystem integrieren. Aber die Adaption des Films und der Massenmedien allgemein für Erziehungszwecke würde ein vollständiges Umdenken von seiten der zuständigen Verwaltung und der Erzieher erfordern, sowie eine vollständige Revision der Erziehungsinhalte. Genau dies illustriert Marshall McLuhans Feststellung: Das Medium ist die Botschaft. Eine Veränderung des Erziehungsmediums bedeutet eine Veränderung der Erziehung selbst. “11

Auch im kirchlichen Bereich kann der Film nicht einfach als zusätzliches, willkommenes - weil zeitgemäßes - Erziehungsmittel übernommen werden. Daß der Einsatz eines Mediums Konsequenzen für die übermittelte Botschaft hat, zeigt sich besonders unter den Bedingungen eines Entwicklungslandes. Das Vorführen eines Films in ländlichen Entwicklungsregionen bedeutet ein Stück Akkulturation, wodurch das traditionelle Normengefüge nachhaltiger erschüttert wird als durch die direkte Vermittlung christlich-abendländischer Wertvorstellungen. Angesichts der so bedingten Orientierungslosigkeit besteht die Gefahr, daß die Rezipienten der Suggestivkraft des neuen Mediums erliegen, was, besonders im Fall des religiösen Films, nicht die Freiheit und Selbstentfaltung, sondern die Abhängigkeit fördert. Unter dieser Rücksicht müßte die kirchliche Filmarbeit in Entwicklungsländern von den für die Seelsorge Verantwortlichen stärker als bisher reflektiert werden. Die religionspädagogische Reflexion müßte konkret etwa von folgenden Fragen ausgehen: 
1. Besteht bei den Teams der Kinomobile sowie bei den diözesanen, regionalen oder nationalen kirchlichen Kommissionen für Kommunikationsförderung Klarheit über die spezifisch pädagogische Zielsetzung der einzelnen Vorführung oder eines Zyklus von Filmveranstaltungen?

2. Handelt es sich bei den angestrebten Zielen um globale, prinzipiell nicht überprüfbare Ziele (Glaube, Bekehrung, humanitäre Einstellung) oder um operationalisierte Ziele (überprüfbares Wissen, registrierbares Interesse, nachweisbare Aktionen)? ${ }^{32}$

3. Ist der Zusammenhang von Ziel und eingesetzten Mitteln durchsichtig?

4. Sind Effektivitätskontrollen vorgesehen?

5. Besteht die Möglichkeit und die Bereitschaft, aus dem „feedback“ von Zielsetzung und tatsächlicher Effizienz Konsequenzen für die Planung einerseits und den Einsatz der Mittel andererseits zu ziehen, oder rät die oft beobachtete Tendenz, daß einmal begonnene pastorale Projekte sich verselbständigen und unabhängig von ihrer Effizienz gerechtfertigt werden, von ihrem probeweisen Einsatz ab?

\section{Perspektiven für die Arbeit kirchlicher Kinomobile}

Aus den kommunikationstheoretischen und religionspädagogischen Überlegungen könnten etwa folgende Konsequenzen für den Einsatz von Kinomobilen der Kirche in Entwicklungsländern gezogen werden:

1. Grundsätzlich kann man davon ausgehen, daß der Film durch die besondere Wirkung des bewegten Bildes geeignet ist, die Menschen in Entwicklungsländern schnell und intensiv zu Innovationen anzuregen. In diesem Zusammenhang ist auf die medienspezifische Verwendungsweise von Film und Tonbild zu verweisen: Während der Film eher die Gesamtpersönlichkeit des Zuschauers anspricht und eine Motivierung des Menschen über die Empfindungskraft auslösen kann, eignet sich das Tonbild eher für didaktische $Z$ wecke im engeren Sinn, etwa für Information, Demonstration oder Repetition.

2. Der Film wird nur in Zusammenarbeit mit anderen Kommunikationsformen (Literatur, Katechese, persönliches Gespräch, Sozialarbeit, Tonbild) wirksam. Das visuelle Erlebnis bedarf einer sachgerechten Entschlüsselung durch Priester, Katecheten oder Entwicklungshelfer. ${ }^{13}$

3. Die Emotion ist als solche noch kein Kriterium für die beabsichtigte Wirksamkeit des Films. Die - freilich schwer zu bestimmende - Zielsetzung kirchlicher Arbeit mit Kinomobilen könnte folgendermaßen umrissen werden: Mindestens einem Teil der Zuschauer müßte zu Bewußtsein kommen, daß mehr Gerechtigkeit und Frieden, als Chriffren für sozialen Fortschritt und Erlösung ${ }^{14}$, notwendig, möglich und erstrebenswert sind.

4. An Inhalt und Form der von kirchlichen Kinomobilen gezeigten Filme sind bestimmte Anforderungen im Sinne der oben zitierten Unesco-Studie von P. Hopkinson zu richten.

5. Ein gezielter Einsatz von Kinomobilen ist nur im Rahmen eines Strukturplanes für die Pastoralarbeit möglich. Bei isolierter Verwendung von Kinomobilen, etwa aufgrund des besonderen Engagement eines Missionars, steht und fällt das Projekt mit der Eignung und Verfügbarkeit einer einzelnen Person. Die Verwirklichung einer Kommunikation im Medienverbund wird, wie einige Erfahrungen zeigen, durch 
solche punktuelle Aktivität erschwert, die Strategie gerät in die Gefahr der Beliebigkeit. Demgegenüber hätte ein Pastoralplan auf diözesaner, regionaler oder nationaler Ebene aufzuzeigen, wie sich die Arbeit der Kinomobile zu anderen audio-visuellen Mitteln, zu anderen Massenmedien, $\mathrm{zu}$ anderen katechetischen, missionarischen und sozio-ökonomischen Tätigkeiten der Kirche verhält, wie die Kontinuität der Projekte zu sichern ist und durch welche Mechanismen die Effizienz der Arbeit gesteigert werden kann.

6. Vor Beginn neuer Projekte sind die vorhandenen audio-visuellen Mittel, auch die in Betrieb befindlichen Kinomobile, auf ihre rationellere Verwendung hin zu untersuchen. Außerdem sind die Möglichkeiten der Zusammenarbeit mit vergleichbaren Unternehmen anderer Kirchen oder staatlicher Stellen aufzuzeigen.

\section{Anmerkungen:}

1. Stellvertretend dafür sei hier zitiert: A Report on the Meeting of the Directors of the Audio-Visual Mobile Units of Tamilnadu held on 30-31st March 1970 at Catholic Centre Tindinavam, S. Arcot, South India (hektographiert).

2. Auskunft vom 10. 5. 1973.

3. Vgl. A. Report on the Meeting of the Directors of the Audio-Visual Mobile Units of Tamilnadu, a.a.O.

4. Paris 1971, Reports and Papers on Mass Communication, Nr. 64.

5. Vgl. a.a.O., S. 25.

6. Vgl. a.a.O., S. 12, 13, 19-21, 31.

7. Vgl. a.a.O., S. 25.

8. Vgl. a.a.O., S. 29.

9. Vgl. a.a.O., S. 26.

10. Vgl. a.a.O., S. 33-36.

11. G. Roberge: Let the Cinema do what the schools can't - educate the masses. In: „Filmfare ${ }^{\alpha}$, Bombay, May 5, 1972, S. 29.

12. Vgl. G. Stachel: Lernziele und Religionsunterricht. In: Heinemann/Stachel/Vierzig, Lernziele und Religionsunterricht, Zürich-Einsiedeln-Köln 1970, S. 24-29.

13. Vgl. Challenge and Response, re-thinking the responsibility of the Churches in the field of audio-visual media. Lecture by Drs. Jan Hes on the 3rd June, International Ecumenical AVA Consultation. Hedenesse, Cadzand, Netherlands 1972, S. 4.

14. Vgl. W. Kasper: Die Heilssendung der Kirche in der Gegenwart (Pastorale). Mainz 1970.

\section{Weitere Literatur:}

G. Albrecht: Film und Verkündigung. Probleme des religiösen Films. Gütersloh 1962 (Neue Beiträge zur Film- und Fernsehforschung, 2).

G. Roberge: Der Film und seine Bedeutung für Indien. In: „Communicatio Socialis“, 5 (1972), Nr. 3, S. 213-220.

Film im Aufbruch. Eine Dokumentation über Filmentwicklungen in Afrika und Südamerika. Berlin-Leipzig 1966. 


\section{S U M M A R Y}

The use of Mobile Film Units for purposes of development and evangelization is becoming more popular. To insure maximum results and to prevent unwise investments it is necessary to have a theoretical concept that will give its users some kind of guidelines to follow. Taking from a UNESCO study (P. Hopkinson, "The Role of Film in Development", Paris, 1971) and considering catechetical-pedagogical views the following points for the use of mobile film units are given by the author: 1. Films can basically be used in rural areas to stimulate the people through the moving pictures for innovations. 2. But films can not be effective unless inter-personal communication is established. 3. Over and above the emotional effect on the viewer of films the followup has to be geared to an overall development of the viewer leading to more freedom, justice and self reliance. 4. Content and form especially of religious films have to be relevant to the local needs. 5 . The use of mobile film units is going to be more effective in a media system based on pastoral needs than planning them independently of it. 6. Existing means and possibilities for ecumenical cooperation and collaboration with other than Church Institutions must be put to better use.

\section{RÉ S U MÉ}

L'utilisation de cinémobiles à des fins de développement et d'évan-gélisation devient plus populaire. Un concept théorique qui donne aux utilisateurs une sorte de ligne de conduite qu'ils peuvent suivre est nécessaire afin d'atteindre les meilleurs résultats possibles et d'éviter des investitions maladroites. D.'après une étude de I'UNESCO (P. Hopkinson, "The role of the film in development", [Le rôle du film dans le développement], Paris, 1971) et des points de vue pédago-catéchétiques, l'auteur énumère les points suivants en faveur de la mise en place de cinémobiles: 1. Les films peuvent être utilisés en règle générale dans les campagnes afin qu'ils incitent les gens à innover; 2. Mais les films ne peuvent être efficaces que s'il y a communication entre les personnes; 3 . Par delà l'effet émotionel sur ceux qui assistent aux films, le processus doit se faire en direction d'un développement total du spectateur afin qu'il l'amène à plus de liberté, d'égalité et de confiance en soi. 4. Le contenu et la forme des films, surtout des films religieux, doivent prendre au sérieux les besoins locaux. 5. La mise en place de cinémobiles doit se faire de facon efficace dans un système de moyens qui se basent plus sur les besoins pastoraux et ne les laissent pas de côté lors de son établissement. 6. Les moyens existant et les possibilités de coopération et de collaboration oecuméniques avec des institutions autres que celles de l'Eglise doivent être mieux utilisés.

\section{R E S U M E N}

Cada vez tiene mayor aceptación la utilización de cinemóviles con fines de desarrollo y evangelización. Para conseguir resultados óptimos y prevenir incersiones imprudentes se requiere un criterio teórico que ponga en manos de los consumidores una especie de líneas fundamentales a las que atenerse. Según un estudio de la UNESCO (P. Hopkinson, „The Role of Film in Development" [El papel del cine en el desarrollo], Paris, 1971) y a tenor de criterios catequético-pedagógicos, el autor da las siguientes pautas para la utilización de cinemóviles: 1. Las películas pueden ser utilizadas en zonas rurales fundamentalmente con el propósito de interesar a la población en las renovaciones. 2. Pero las películas sólo pueden ser eficaces mediante la comunicación interpersonal. 3. Superando el efecto emocional que el film produce en el espectador, el sistema debe estar dirigido a un desarrollo específico del espectador, a fin de orientarle hacia una mayor libertad, justicia y confianza en sí mismo. 4. El contenido y forma, ante todo de las películas religiosas, deben tomar muy en serio las necesidades locales. 5. El empleo de cinemóviles debe ser eficazmente incorporado a un sistema de medios de comunicación social, que se fundamente en las necesidades pastorales y no deje de tener en cuenta dichas necesidades a la hora de planificar. 6. Deben ser mejor aprovechados los medios de que se dispone y las posibilidades de cooperación ecuménica y colaboración con otras instituciones eclasiásticas. 